\title{
REVUES CRITIQUES
}

\section{LES ASTERINÉES PARASITES DE L'HOMME.}

\section{LA PIEDRA}

\section{Par Maurice LANGERON}

Le terme d'Astérinées ne répond pas à une unité systématique, à une famille végétale ; néanmoins il désigne un groupement très naturel, dans lequel on a réuni des champignons présentant les mêmes particularités biologiques. Ces champignons vivent en parasites sur les feuilles des plantes, à la surface desquelles ils forment des taches généralement épaisses, brunâtres ou noirâtres, analogues aux fumagines de nos pays. Mais ces dernières se développent à peu près exclusivement sur le miellat, enduit sucré excrété par les pucerons et les cochenilles; ce sont donc de simples saprophytes qui végètent partout où tombe le miellat, aussi bien sur des objets inertes, bois, métal ou pierre, que súr les parties vivantes des végétaux. Sur les feuilles, nos fumagines européennes vivent aussi en saprophytes et restent purement superficielles, tandis que les astérinées sont de véritables parasites, dont les suçoirs pénètrent dans les tissus de l'hôte.

Les Astérinées sont rares en Europe (1), parce qu'elles ne rencontrent pas dans notre climat les conditions hygrométriques qui leur sont indispensables. En effet, ces champignons, presqu'entièrement superficiels, ont des besoins d'eau considérables qui ne peuvent être satisfaits que par un climat très humide, tandis que les fumagines d'Europe, vivant sur un substratum sucré et par conséquent hygroscopique, trouvent beaucoup plus facilement l'eau qui leur est nécessaire; toutefois, elles se développent surtout dans les expositions ombragées et humides. En fait, on ne rencontre les Astérinées que dans les régions où il tombe au moins un mètre de pluie par an. Leur distribution géographique est donc subordonnée

(1) Il n'y a en Europe que cinq espèces d'Astérinées : trois Meliola, et deux Microthyriées wardinées, le Dimerosporium veronica et le Questieria pulchra.

AnNales de Parasitologie, T. VII, No $4 .-1^{\text {er }}$ juillet 1929 , p. 309-324. 
à celle des pluies et cette dépendance est si étroite qu'Arnaud (1918) a donné le nom de climat astérinéen au climat très humide des régions du globe où vivent ces champignons. Ces conditions sont réalisées surtout dans les zones tropicales et subtropicales, plus rarement dans les zones tempérées. C'est donc dans la zone intertropicale des deux mondes qu'on rencontre ces champignons et l'Amérique du Sud, particulièrement pluvieuse, surtout dans les bassins de l'Amazone et du Parana, se montre très favorisée. Toutefois, l'aire des Astérinées déborde notablement les tropiques, puisqu'on en trouve des stations en Europe, dans certaines conditions d'humidité, entre $40^{\circ}$ et $60^{\circ}$ de latitude nord. Rien de pareil n'a été observé dans l'hémisphère sud, beaucoup plus froid et où

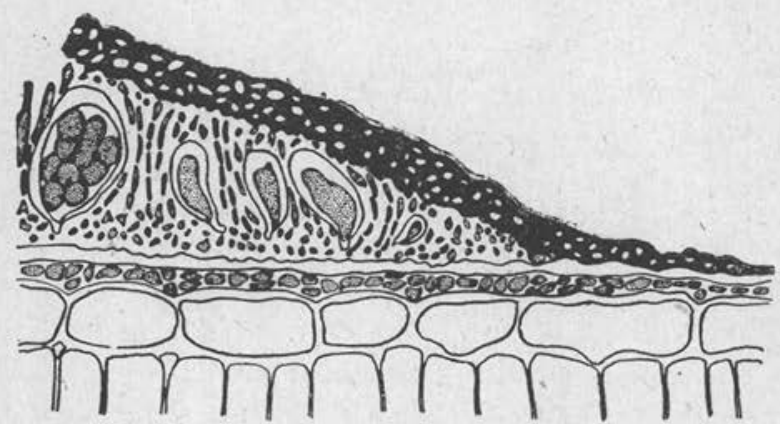

Fig. 1. - Type d'ascostroma de Microthyriacée (Balansina stellata Arnaud) avec ascospores bicellulaires. D'après Arnaud.

les terrés sont bien moins étendues; les Astérinées n'y descendent pas au delà de $40^{\circ}$ de latitude sud.

Arnaud, qui a si bien étudié ce groupe, attribue au climat astérinéen une action morphogénétique faisant en quelque sorte " émerger » le champignon à la surface de l'hôte et donnant ainsi naissance au stroma si caractéristique des Astérinées. Ce stroma, comme l'a montré Arnaud, présente un caractère xérophytique marqué, dû à la nature du substratum dans lequel le champignon ne peut puiser beaucoup d'eau. Il se trouve donc, malgré l'humidité du climat, exposé à des intervalles de sécheresse et de dessiccation. De là résulte l'aspect du stroma qui est compact, cutinisé, formé de filaments mycéliens vermiculés, étroitement soudés et anastomosés entre eux (fig. 1).

La présence de caractères xérophytiques est fréquente chez les plantes hygrophiles vivant sur un sol dur et sec, quoique dans unc atmosphère humide; ełle existe aussi chez les plantes habitant 
les lieux humides, tels que les tourbières et les marais salants. Schimper, pour expliquer ce fait, en apparence parodoxal, a eu recours à l'ingénieuse hypothèse du sol physiologiquement sec qui, bien que saturé d'eau, la retient par suite de la présence d'éléments chimiques particuliers, tels que les acides humiques libres ou les chlorures. Il n'est donc pas surprenant que la convergence épharmonique ait donné des caractères communs au mycélium végétatif des champignons à facies astérinoïde. Il n'en a pas été de

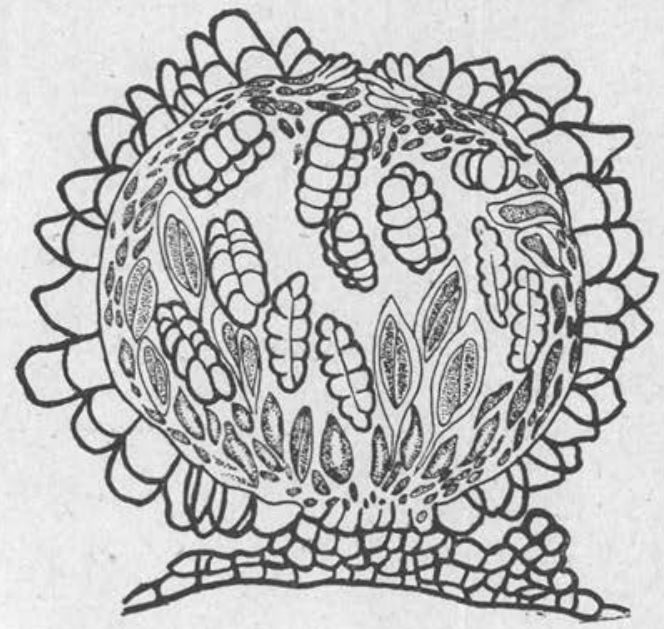

Fı́. 2. - Périthèce ou conceptacle mûr de Meliola sp. (Dothidéale). D'après Arnaud.

même pour les organes reproducteurs, beaucoup moins sensibles aux actions morphogénétiques.

On a donc pu reconnaître que les Astérinées sont pour l'immense majorité des Ascomycètes du groupe des Pyrénomycètes, c'est-àdire dont les périthèces sont des conceptacles pourvus d'une petite ouverture ou ostiole (fig. 2 et 3 ). Toutefois, chez les champignons à facies astérinéen, le conceptacle n'est pas toujours aussi net que chez les autres pyrénomycètes. Les asques naissent souvent dans des loges sans paroi propre qui se creusent dans le stroma (fig. 1). Arnaud a donné à ce mode primitif de fructification le nom d'ascostroma (fig. 1): on l'observe chez les Microthyriales (1) qui forment

(1) Les Pyrénomycètes (champignons à noyau ou périthèce) sont des ascomycètes dont les asques se développent à l'intérieur d'un fruit ou périthèce pourvu d'une ouverture ou ostiole, par consêquent déhiscent. Cette définition s'oppose à celles des Gymnoascées qui ne forment pas de fruit et dont les asques sont libres, des Périsporiacées dont le périthèce fermé est indéhiscent et des Discomycètes dont les asques naissent sur un plateau ou disque.

L'hymenium, qui fait défaut chez les Gymnoascées et les Pérosporiacées, com- 
une grande partie des Astérinées avec de nombreux genres et espèces. Les véritables périthèces (fig. 2) se rencontrent chez d'autres types, voisins des Dothidéales et représentés surtout par les Meliola,

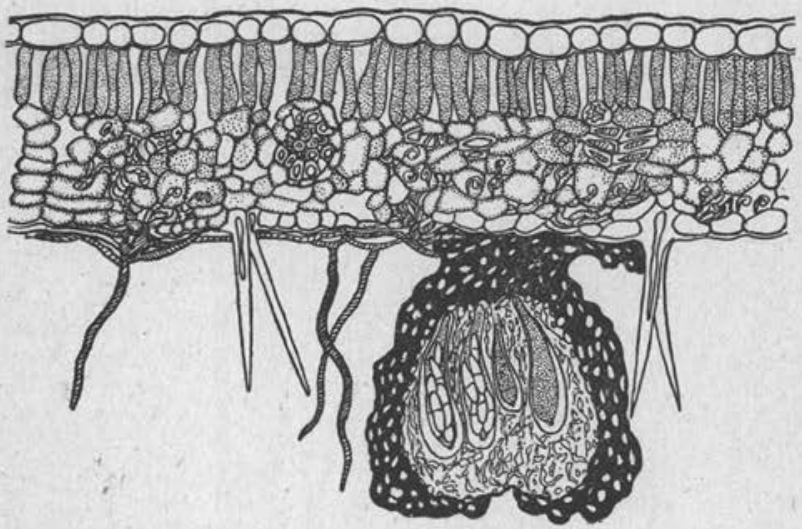

Fig. 3. - Périthèce libre de Parodiopsis melioleides (Winther) Maubl. (Hypocréale). Sur Pera leandri, Porta das Caixas, Rio de Janeiro. D'après Arnaud.

dont on connaît environ 300 espèces ; bien entendu, les champignons astérinoïdes qui se rattachènt aux Sphériales et aux Hypocréales (fig. 3) présentent aussi des périthèces typiques. 'Toute-

mence à apparaître chez les Pyrénomycètes dont les périthèces sont tapissés d'asques disposés parallèlement et entremêlés de paraphyses; il se développe pleinement chez les Discomycètes, où il recouvre toute la surface du disque.

Le thalle des Pyrénomycètes est formé de filaments ramifiés et cloisonnés présentant une grande tendance à s'accoler en massifs, plaques ou cordons qui forment des pseudo-parenchymes ou stromas, dont la surface est souvcnt cutinisée et fortement colorée. Suivant cette coloration, on peut donc, avec Arnaud, diviser les Pyrénomycètes, en deux séries: l'une colorée en brun (Phaeopyrénomycètes) comprenant les Sphériales, les Microthyriales et les Dothidéales; I'autre (Calopyrénomycètes) colorée en rouge ou autres teintes vives, comprenant surtout les Hypocréales.

Les Microthyriales sont voisins des Myriangiales, type primitif des Pyrénomycètes stromatiques : dans ce type, les asques naissent immergés et dispersés dans le tissu du stroma et non localisés dans des loges comme chez les Pyrénomycètes vrais. Chez les Micróthyriales, les asques se forment déjà dans des zones particulières du stroma ascigère (ascostroma d'Arnaud). Ces cavités ou loges sont beaucoup moins bien délimitées que chez les Dothidéales et surtout les Sphériales ; elles sont remplies de cellules gélifiées ou de tissu paraphysoide; les ascospores sont typiquement bicellulaires. La surface des stromas présente une structure radiaire, à filaments parallèles à la surface de l'hôte.

Chez les Dothidéales au contraire, le stroma est formé par des filaments dressés parallèlement vers l'extérieur; il est plus épais et les loges sont mieux limitées. Arnaud y rattache les Meliola et Amazonia qui ont un facies astérinoïde et dont les premiers possèdent des périthèces ou conceptacles libres naissant d'un stroma rayonnant (fig. 2).

Chez les Sphériales, les stromas et les périthèces sont de couleur très foncée, carbonacés. Le périthèce est libre ou immergé et possède en général une paroi 
fois, chez certaines Microthyriales, on n'a pas observé d'asques, mais seulement des pycnides (1).

Il faut retenir en outre que chez les Astérinées microthyriales, la structure du stroma est radiaire (fig. 15 à 18), tandis que chez les Astérinées dothidéales, les filaments mycéliens sont dressés parallèlement vers l'extérieur ; cette distinction n'est d'ailleurs pas toujours absolue.

Notons enfin qu'un certain nombre d'Astérinées sont hyperparasites sur d'autres Astérinées.

En résumé, les Astérinées sont des champignons pyrénomycètes vivant à la surface des feuilles dans les climats très humides, à pluies très abondantes, de préférence dans la zone intertropicale. Ces champignons forment des taches épaisses, brunes ou noires, coriaces, constituées par un pseudoparenchyme ou stroma, entouré de filaments mycéliens. Dans la masse du stroma, se forment des conceptacles ou périthèces plus ou moins bien différenciés.

Jusqu'ici, nous n'avons rencontré que des espèces parasites des plantes. Jamais, à ma connaissance, aucune astérinée n'a été signalée comme parasite de l'homme dans les régions à climat astérinéen. Pourtant, lorsqu'on étudie les caractères morphologiques et la distribution géographique de la piedra, on est frappé des analogies que présentent les champignons qui produisent cette maladie nodulaire des poils avec les champignons astérinéens.

La piedra est surtout connue en Amérique du Sud où elle a été signalée en Colombie, au Brésil et au Paraguay. Or les localités où cette affection existe, se trouvent précisément dans les régions a climat astérinéen, où il tombe de un à deux mètres de pluie par an. En Colombie, Medellin et Antoquia, les deux principaux foyers de piedra, se trouvent dans une zone à deux saisons pluviales, avec un à deux mètres de pluie par an et plus de deux mètres sur le versant pacifique. Au Brésil, la piedra est connue depuis Bahia, qui se trouve à l'extrémité nord de la zone littorale atlantique où il tombe plus de deux mètres de pluie par an, jusqu'à Santos, tout le long de la Serra do Mar, région de roches cristallines avec croupes et mornes et couverte d'une forêt tropicale littorale. Cette zoné

propre, mais on trouve tous les intermédiaires, entre ce groupe et les précédents. Les Sphériales renferment de nombreuses formes astérinoïdes ou hyperparasites d'Astérinêes.

Le périthèce et le stroma des Hypocréales sont mous et de couleur claire : ces champignons forment en somme une série parallèle à celle des Sphériales. Les périthèces peuvent être libres ou immergés. Des champignons de ce groupe vivent fréquemment en hyperparasites sur les Astérinées et prennent le facies astérinoïde.

(1) Les pycnides sont des conceptacles qui produisent non des asques, mais des conidies ou stylospores. 
est tout à fait celle du climat astérinéen ; les Astérinées y prospèrent. En outre, la piedra a été signalée dans le centre du Brésil, à Cuyaba, dans le Matto Grosso, encore dans une zone à pluies abondantes et non loin des grands marécáges du Haut-Paraguay ; puis à Bello-Horizonte, dans la Serra d'Espinhaço, toujours dans une zone forestière et humide.

Au Paraguay, la piedra a été signalée à Asuncion par Migone et Cavazzoni, puis par Delamare et Gatti (1928). Ce pays se trouve dans la zone de répartition des Astérinées et la région qui avoisine le confluent du Parana et de l'Iguazu reçoit mêméplus de deux mètres de pluie par an.

En ce qui concerne les caractères morphologiques des champi-

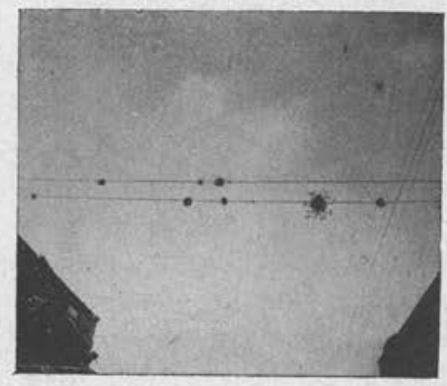

FIg. 4. - Touffes de Broméliacées (Tillandsia) sur des fils électriques aériens à Perico (Rép. Argentine). Cliché M. Langeron.

gnons de la piedra, je ne parlerai que de ceux des piedras brésilienne et paraguayenne qui seuls sont bien connus. Je n'ai pas vu de piedra colombienne et les descriptions qui en ont été données sont très incomplètes. Nous verrons cependant plus loin qu'il est peut-être possible d'en faire état.

P. Parreiras Horta a publié, en 1911, une description de la piedra brésilienne qui restera comme un modèle d'observation minutieuse et précise. La valeur en est d'autant plus grande qu'elle a été faite d'une manière absolument objective et sans aucune idée préconçue. Aussi E. Brumpt a-t-il consacré cette valeur en dédiant à P. Horta, sous le nom de Trichosporum hortai Brumpt, 1913, le champignon de la piedra brésilienne. L'interprétation proposée par Horta pour les faits qu'il avait constatés se trouve parfaitement justifiée et exacte. Il est surprenant qu'elle n'ait pas reçu plus tôt une éclatante confirmation de la part des mycologues. Je vais tenter de combler cette lacune et de faire ressortir, à la lumière de la mycologie comparée, la justesse des vues de P. Horta. 
J'ai déjà montré que la piedra sud-américaine se trouve dans des zones à climat astérinéen. Sous ce climat, l'atmosphère est si humide que les fils électriques aériens eux-mêmes ont leur piedra, sous forme de nodules globuleux constitués par des touffes d'une

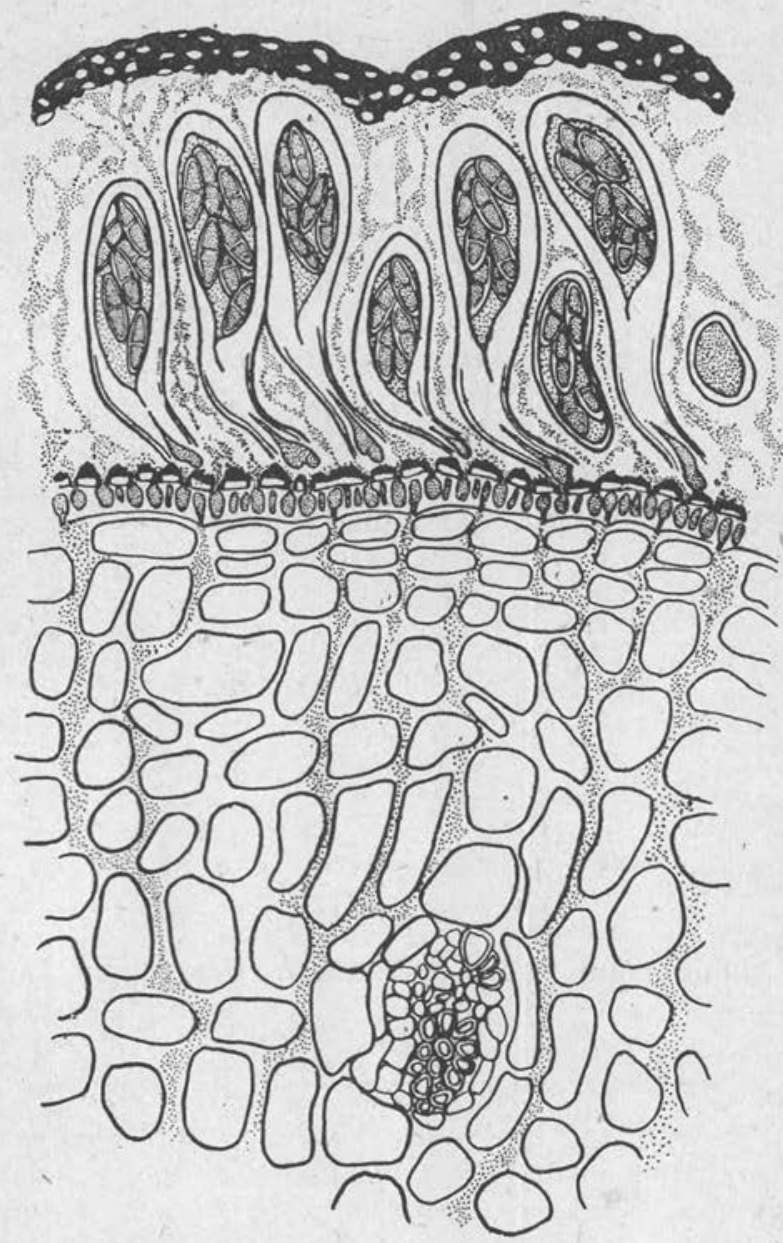

Fig. 5. - Ascostroma de Protothyriée (Protothyrium salvadoræ)(Cooke) Arnaud. D'après Arnaud.

petite broméliacée du genre Tillandsia (fig. 4). On n'est pas pelı surpris de voir ces épiphytes végéter sur un substratum aussi pauvre. On comprend aussi pourquoi ces plantes, si exposées à la dessiccation, présentent des caractères xérophytiques très nets, ce qui est aussi le cas des Astérinées. 
Pour les champignons de la piedra, l'analogie ne s'arrête pas là. L'examen des nodules piédriques montre un stroma exactement comparable à celui des Astérinées, entouré à la périphérie d'une frange de filaments mycéliens (10, fig. 14). Il s'agit bien ici d'un ascostroma, car les kystes si bien décrits par Horta ne sont autres que des asques remplis d'ascospores. Il y a bien eu à ce sujet quelques confirmations timides et rêticentes, mais personne, à ma connaissance, n'a encore parlé de l'analogie pourtant si évidente entre les kystes d'Horta et les asques des Astérinées.

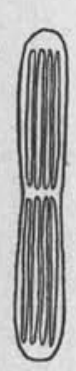

a

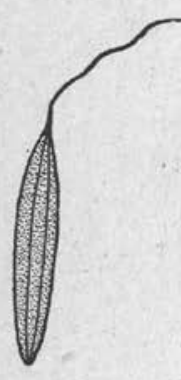

b

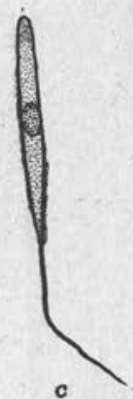

FIg. 6. - Ascospores (scolécospores) de Nematospora coryli Peglion (Saccharomycétacée); $a$, asque libre avec ses deux groupes de quatre scolécospores; $b$, groupe de trois scolécospores; $c$, une scolécospore isolée et colorée. D'après Peglion.

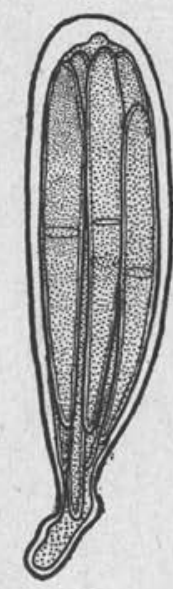

Fig. 7. - Asque et ascospores (scolécospores) de Parodiopsis sweetia (P. Henn.) Arnaud. (Hypocréale). D'après Arnaud.

Les asques du Trichosporum hortai ne paraissent pas groupés dans des loges, mais semblent naître isolément dans la masse du stroma. Il n'y aurait done pas de véritables conceptacles, mais une structure analogue à celle qu'Arnaud a décrite pour les Protothyriées (fig. 5), qui réalisent le type le plus simple des Microthyriacées. Ce caractère indique nettement la place que doit occuper le champignon de la piedra brésilienne dans la classification mycologique. Pourtant on ne peut placer ce champignon dans le genre Protothyrium Arnaud, 1917, à cause de la forme de ses ascospores. En effet, les ascospores du Protothyrium salvadoræ (Cooke) Arnaud sont longuement ovoïdes et bicellulaires (fig. 5), tandis que celles des kystes d'Horta (12, fig. 14), sont du type scolécospore ou spores vermiformes (de $\sigma \times(\dot{\omega}) \eta \eta \xi$, ver), c'est-à-dire en forme de fuseau allongé, terminé à chaque extrémité par un filament. Horta décrit ces ascospores comme non cloisonnées. 
Les scolécospores sont fréquentes chez les ascomycètes et sont réparties dans des groupes très différents. C'est ainsi que le Nematospora coryli Peglion, 1901, est une levure découverte en 1897 par Peglion dans des noisettes et dont les asques renferment deux groupes superposés de quatre ascospores (fig. 6) semblables à celles qui ont été figurées par Horta, mais à un seul filament polaire. Le

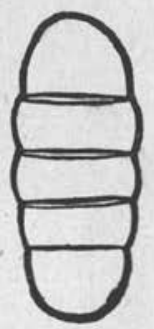

Fig. 8. - Ascospore multiloculaire d'Amazonia psychotrix (P. Henn.) Th. (Dothidéale). D'après Arnaud.

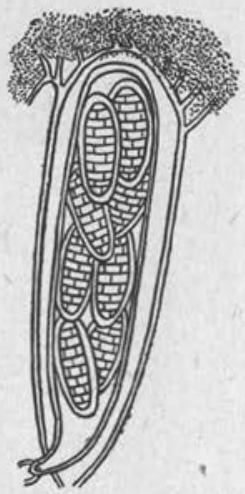

Fig. 9. - Asque, ascospores murales et paraphyses d'Hysteropsis culmigena Rehm. (Hystériinée). D’après Rehm.

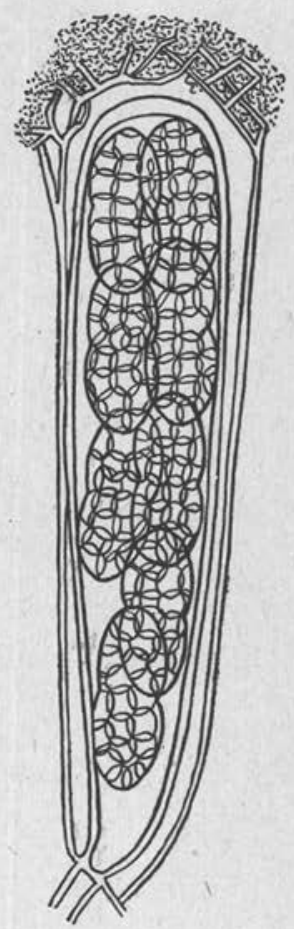

Fig. 10. - Asque, ascospores murales et paraphyses d'Hysterographium fraxini (Pers.) de Not. (Hystériinée). D'après Rehm.

Parodiopsis sweetiæ (P. Henn). Arnaud, qui est une hypocréacée, a des ascospores vermiformes à deux loges et un seul filament (fig. 7). La grande majorité des Microthyriacées donne des ascospores ovoïdes à deux loges (fig. 1), tandis que celles des Meliola (fig. 2) et genres voisins (fig. 8) ont 5-6 loges. Cependant les Scolecopeltis, qui sont des Microthyriacées, ont des scolécospores.

Le polymorphisme des ascospores parait donc être la règle chez les Ascomycètes. On pourrait en citer de nombreux exemples. Ainsi, 


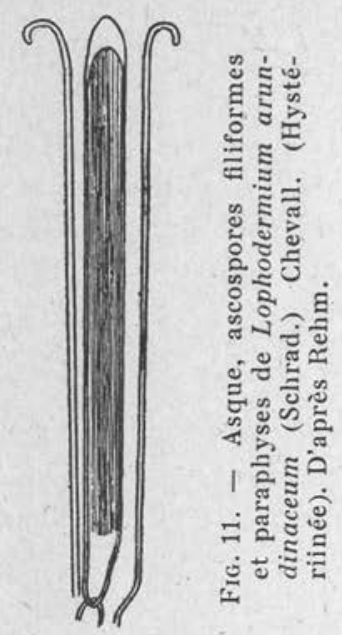

chez les Hystérinées, on voit les ascospores murales des Hysteropsis (fig. 9) ou des Hysterographium (fig. 10) voisiner avec les ascospores si longuement filiformes des Lophodermium (fig. 11), des Lophium (fig. 12) et des Acrospermum. Chez les Phacidiinx, les Rhytisma (B, fig. 13) ont des ascospores longuement fusiformes, tandis que celles du genre voisin Pseudorhytisma (A, fig. 13) sont brièvement ovoïdes, etc., etc.

Il en est de même pour les paraphyses (1) (fig. 9, 10,11,12,13). Ces organes manquent dans beaucoup de genres de Microthyriacées ; il n'est donc pas surprenant qu'elles n'existent pas autour des asques du Trichosporum hortai.

Les particularités si bien observées et décrites par Horta pour la piedra brésilienne ont été retrouvées dans la piedra paraguayenne par Delamare et Gatti. Leur description confirme celle d'Horta et la complète pour quelques points. Ces auteurs semblent avoir vu l'articulation des asques avec les cellules du pseudoparenchyme de l'ascostroma ; les filaments ascogènes n'ont pu être définis, mais l'asque paraît naître aux dépens d'une cellule qui augmente de volume et devient piriforme. Son noyau se divise, tandis que le protoplasma devient granuleux et finalement les ascospores s'individualisent au nombre de 6,8 ou 12 . Comme Horta l'avait déjà figuré (1 à 8 , fig. 14), leur développement les amène à atteindre les deux pôles de l'asque, puis, la croissance continuant, elles se pelotonnent et replient leurs filaments polaires, tout en restant parallèles au grand axe de l'asque
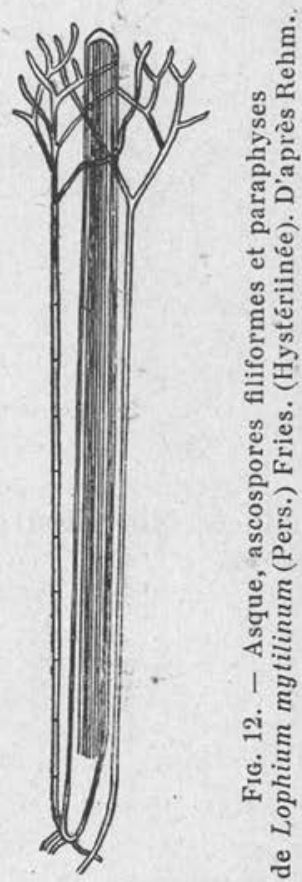

(1) Les paraphyses sont des cellules végétatives stériles, en forme de poils plus ou moins compliqués, qui sont entremêlées aux asques et aux basides dans le tissu fertile ou hyménium. Les périphyses sont aussi des filaments stériles mais qui tapissent seulement la paroi interne du périthèce, słans avoir de rapport avec l'hymenium; ils peuvent s'allonger au point de sortir par l'ostiole et de fermer le col du périthèce. 
(8 et 9, fig. 14). Delamare et Gatti ont vu parfois une cloison dans les ascospores.

Il n'a pas été signalé d'ostioles à la surface de l'ascostroma ; pourtant les ascospores sont facilement mises en liberté, soit par suite des manipulations auxquelles sont soumis les nodules pendant l'examen dans la potasse ou le lactophénol, soit par simple action de l'eau. Dans ce dernier cas, il se produit un phénomène normal chez les Pyrénomycètes : c'est la dissolution des cellules

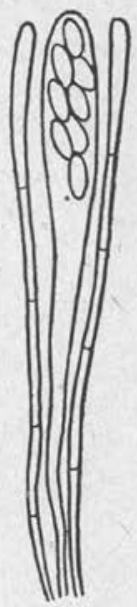

$\boldsymbol{A}$

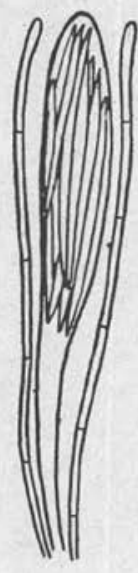

$B$

FIg. 13. - Asque, ascospores et paraphyses: A, de Pseudorhytisma bistorta (D. C.) Juel. ; B, de Rhytisma acerinum (Pers.) Fries. (Phacidiinées.) D'après Lindau.

gélifiées qui entourent les asques et constituent des loges plus ou moins parfaitement différenciées. Après la déhiscence de l'asque, les ascospores cheminent vers l'extérieur, à travers ces cellules gélifiées et sont expulsées par suite de leur gonflement. Le mécanisme de ce phénomène demanderait à être précisé par de nouvelles observations.

Delamare et Gatti ont été moins heureux dans l'interprétation du mycélium qui se trouve dans la zone d'accroissement, à la périphérie du nodule. Ils décrivent des conidies là où il y a simplement accroissement et allongement des filaments mycéliens par di et trichotomie, phénomène tout à fait normal chez les Astérinées dont les ascostromas s'étendent toujours sous forme de disques, par suite de ramifications multiples. C'est ce qu'Arnaud a figuré chez les Balansia (fig. 15), Hariotula (fig. 16), Morenoina, Microthyrium 
(fig. 17), Prillieuxina, etc. Cette disposition correspond à la frange des Microthyriacées (fig. 16 et 17) qui joue le rôle de crampon et assure l'adhérence au support. Cette frange disparaît dans les types

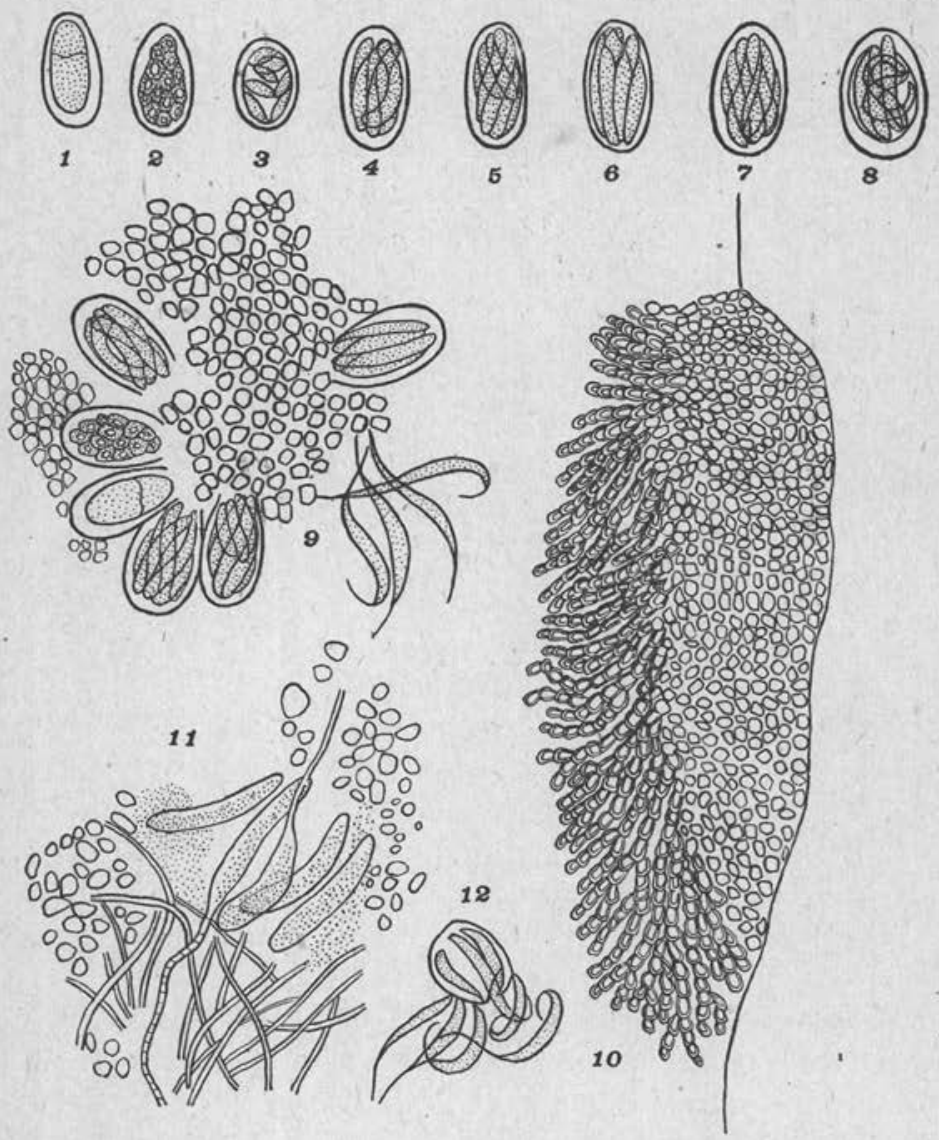

Fig. 14. - Piedraia hortai (Brumpt) da Fonseca et Leao. 1 à 8 , développement des ascospores (scolécospores); 9 , asques à divers stades et scolécospores isolées dans l'ascostroma a près dissociation ; 10 , ascostroma à la surface du cheveu ; 11 , germination des scolécospores; 12 , déhíscence de l'asque et mise en liberté des scolécospores. D’après Horta.

hyperparasites (Trichothyrium, fig. 18), chez lesquels l'ascostroma repose, non sur la surface de la feuille, mais sur un autre champignon, le plus souvent astérinoïde

Il semble donc que la piédra paraguayenne est très semblable, sinon identique, à la piédra brésilienne. Cette question sera facile- 
ment tranchée par la mensuration comparée des éléments des deux parasites, car les petites différences culturales mentionnées par Delamare et Gatti peuvent être attribuées à de simples variations dans les milieux de culture. Le voisinage des deux pays rend très vraisemblable l'extension de la maladie de l'un à l'autre.

Le problème est beaucoup plus ardu pour la piédra colombienne

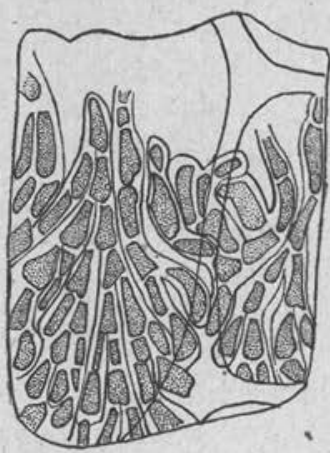

FıG. 15. - Ramifications multiples à la périphérie de l'ascostroma chez $\mathrm{Ba}$ lansina stellata Arnaud. (Microthyriacée). D'après Arnaud.

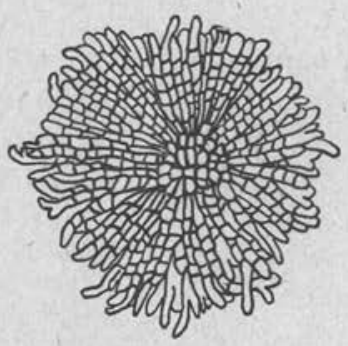

Fig. 16. - Ascostroma étoilé et frangé d'Hariotula loranthi (Karst. et Har.) Arnaud. (Microthyriacée). D'après Arnaud.

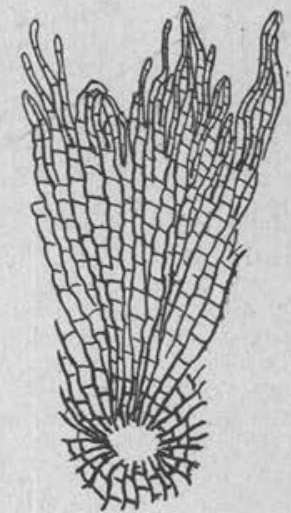

Fig. 17. - Portion de l'ascostroma d'un Microthyrium (M. microscopicum Desm.) (Microthyriacée) montrant la frange. D'après Arnaud.

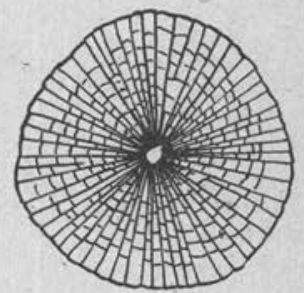

Fig. 18. - Ascostroma non frangé de Trichothyrium fimbrialum Speg., (Microthyriacée) hyperparasite sur un Meliola. D'après Arnaud.

sur laquelle nous possédons peu de documents précis. Il est fort difficile de savoir s'il s'agit ici d'une Astérinée. Les nodules ne seraient pas noirs comme ceux de la piédra brésilienne et la question de la présence des kystes d'Horta, c'est-à-dire des asques, n'est pas encore élucidée. On en est en somme réduit à la description de Desenne, qui date de 1878 , c'est-à-dire de 50 ans. Desenne a parfaitement vu la mosaïque du stroma, formée de grands éléments 
polygonaux, de 12-15 $\mu$ de diamètre. Dans l'épaisseur du stroma, il signale des lacunes contenant « une ou plusieurs grosses cellules incolores qui sembleraient être des thèques (?) 》. Il ajoute que rien ne l'autorise à parler de la déhiscence de ces cavités. Én l'absence de figures, il est impossible de rien décider, mais la description de Desenne, malgré ses réticences, donne bien l'impression qu'il s'agit encore iei d'un ascostroma. Les cultures crémeuses et vermiculées obtenues par Juhel-Renoy et Lion ne peuvent servir à trancher la question, ear il s'agit probablement d'une impureté blastosporée. On obtient quelquefois, en effet, des cultures analogues avec les piédras brésilienne et paraguayenne (Horta, da Fonseca et Leão, Delamare et Gatti). Tout reste donc à faire pour la piédra colombienne, le seul caractère précis qu'on possède à son sujet étant la grande dimension des éiéments superficiels du stroma.

Par contre, il est certain que les piédras ou trichospories européenne et asiatique sont d'origine toute différente et n'ont aucun rapport avec les Astérinées. La description du Trichosporum beigeli (Rabenhorst, 1867), donnée par Vuillemin (1902), reste classique et le genre Trichosporum (Behrend) Vuillemin, doit être conservé pour la plupart des agents de ces trichospories. Il y a toutefois des exceptions. Ainsi, le cas observé à Genève par Ch. Du Bois, sur les poils de la région génitale d'une femme, diffère tout à fait des autres cas de trichospories et était certainement dû à un Monilia, comme le montrent les excellentes planches qui accompagnent ce mémoire. Il est vrai que la localisation est ici exceptionnelle et favorable, à cause de la chaleur et de l'humidité de la région, à la fixation sur les poils d'un blastosporé, développé grâce à la présence du sucre dans l'urine de la malade.

Les trichospories asiatiques ont été étudiées surtout au Japon. Les travaux récents de Kambayashi et d'Ota montrent qu'il s'agit bien encore de véritables Trichosporum.

Il n'est donc plus possible de laisser dans le genre Trichosporum les parasites des piédras astérinéennes. Précisément, O. da Fonseca et Leão (1928) viennent de publier une note très courte mais fort intéressante sur la piédra brésilienne. Eux aussi ont retrouvé les kystes d'Horta, et, sans toutefois en donner d'interprétation, ils pensent que la présence de ces kystes suffit pour justifier la création d'un genre spécial. Nous avons vu que Brumpt, pressentant l'importance des kystes d'Horta, avait donné au champignon de la piédra brésilienne le nom de Trichosporum hortai. Da Fonseca et Leão proposent de créer le genre Piedraia dont l'espèce type sera Piedraia hortai (Brumpt, 1913). La création de ce nouveau genre 
nous paraît très opportune, car elle répond au desideratum que nous formulions plus haut. Ce genre renfermera donc les parasites astérinéens des piédras brésilienne et paraguayenne. Il doit prendre rang parmi les ascomycètes pyrénomycètes, dans le groupement biologique des Astérinées, l'ordre des Microthyriales et la famille des Microthyriacées, au voisinage du genre Protothyrium Arnaud et de la tribu des Protothyriées.

Ces parasites, de même que les véritables Trichosporum, sont très éloignés des dermatophytes, tant par leurs caractères morphologiques que par leur pouvoir pathogène très faible. Les comparaisons qui ont été établies entre ces deux groupes de champignons (Delamare et Gatti) n'ont donc pas de raison d'être. Chez les dermatophytes, les appareils reproducteurs n'apparaissent que dans les cultures ; c'est l'inverse chez les Piedraia, dont la forme ascosporée est exclusivement parasitaire, les cultures ne reproduisant qu'un stroma stérile. Les dermatophytes sont de véritables parasites pathogènes, pénétrant dans l'épiderme et les poils, les envahissant et produisant des lésions variées, locales ou mêmes générales. Les Piedraia se comportent bien plus en épiphytes (ou mieux épitriches) qu'en parasites; leur nutrition est empruntée beaucoup plus au milieu extérieur qu'au support. Celui-ci reste intact ; c'est à peine si l'épidermicule du poil est soulevé et dissocié au point d'implantation du nodule. L'adhérence au poil est surtout assurée par la frange marginale de l'ascostroma. Un point important à étudier sera de savoir s'il existe des suçoirs ou quelqu'organe analogue, comme chez les Astérinées, parasites des végétaux. Ces suçoirs, extrêmement délicats, ont été longtemps méconnus et leur existence a été démontrée par R. Maire.

La connaissance des ascospores des Piédraia et de leur mise en liberté si facile au contact de l'eau (et probablement de la sueur), permet d'expliquer la propagation de la piédra. On conçoit très bien comment les ascospores peuvent contaminer successivement les cheveux d'un même individu et passer d'un individu à l'autre par contact, ce qui explique les épidémies familiales et scolaires. Horta a vu les ascospores se développer sur les milieux artificiels ; leurs filaments terminaux donnent naissance à un mycélium cloisonné (11, fig. 14).

\section{RÉsumé}

Dans les pages qui précèdent, on montre les analogies morphologiques et biologiques qui existent entre les Astérinées parasites des plantes et les champignons qui produisent les piédras brési- 
lienne et paraguayenne. Ce rapprochement permet d'interpréter les kystes découverts par Horta comme des asques renfermant des ascospores du type scolécospore et de considérer les nodules piédriques comme les ascostromas d'une Astérinée d'un type pyrénomycète très primitif, voisin des Protothyriacées. Ces champignons sont donc très différents de ceux qui produisent les piédras ou trichospories européenne et asiatique ; ces derniers ne présentent pas les caractères des Astérinées et doivent rester dans le genre Trichosporum (Behrend, 1890) Vuillemin, 1902. Pour les types astérinéens sud-américains, il y a lieu d'adopter le genre Piedraia, proposé par da Fonseca et Leão (1928). La position de la piédra colombienne, maladie encore insuffisamment connue, reste incertaine.

\section{BiBLIOGRAPHIE}

Arnaud (G.). - Les Astérinées. Thèse de Paris, Doctorat ès sc. nat., 1918, 288 p., $53 \mathrm{pl}$.

Brumpt (E.). - Précis de parasitologie, 2॰ édition, Paris, Masson, 1913 ; cf. p. 951. Delamare (G.) et Gatti (C.). - Sur la piedra du Paraguay. Bull. Acad. de méd., XCIX, 1928, p. 500-503.

- L'évolution des kystes de la piedra paraguayenne. C. R. Acad. des sc., CLXXXVI, 1928, p. 1771-1772.

- Culture de la piedra paraguayenne, C. R. Soc. biol., XCIX, 1928, p. 14251426.

- La piedra del Paraguay. Ann. Fac. ciencias med. Asuncion, II, 1928, p. $10-33,3 \mathrm{pl}$.

- Caractère néoendothrix de la piedra paraguayenne. C. R. Soc. biol., C, 1929, p. 122-124.

Desenne. - Sur la piedra, nouvelle espèce d'affection parasitaire des cheveux. C. R. Acad. sc., 1878.

Du Bois (Ch.). - Etude d'un cas de trichosporie. Ann. dermat. et syphil., 1910 p. $447-456,3 \mathrm{pl}$.

Da Fonseca (O.) et Leáo (A. E.). - Sobre os cogumelos da piedra brasileira. Suppl. Mem. Inst. O. Cruz, no 4, 1928, p. 124-127, 1 pl.

Honta (P.). - Sobre uma nova forma de piedra. Mem. Inst. O. Cruz, III, 1911, p. $86-107$, pl. V-VI.

Lombardo (C.). - Sulla piedra nostras. Giorn. ital. malatt. vener. e pelle, XLV, 1904, p. 308-330, 3 pl.

Maire (R.). - Les suçoirs des Meliola et des Asterina. Annales mycologici, VI, 1908, p. 124-128.

OTA (M.). - Sur quelques champignons pathogènes du type Trichosporum beigeli Vuillemin. Ann. de parasit., IV, 1926, p. 1-13.

Peglion (V.). - Ueber die Nematospora coryli Peglion. Ctrlbl. für Bakt., 2 Abt., VII, 1901, p. 754-761.

Vuillemin (P.). - Trichosporum et trichospories. Arch. de parasit, V, 1902, p. 38-66. 